\title{
Cardiac arrhythmia in refrigerator repairmen exposed to fluorocarbons
}

\author{
C Edling, C-G Ohlson, G Ljungkvist, Å Oliv, Bror Söderholm
}

\begin{abstract}
A field study of 89 refrigerator repairmen was carried out to ascertain whether occupational exposure to fluorocarbons induces cardiac arrhythmia. The concentrations of fluorocarbons in the breathing zones and the heart activity were recorded simultaneously. Most cooling systems contained FC 12 or FC 22. The highest level recorded in one minute was $14000 \mathrm{ppm}$ and the highest time weighted level during eight hours was $280 \mathrm{ppm}$. Two types of arrhythmia were recorded, ectopic beats and sudden bradycardia. A within subject comparison design was applied and the main parameter was the difference in arrhythmia frequencies between exposed and unexposed periods. No appreciable differences between exposed and unexposed periods and no consistent dose effect relations were observed, although subjects in the medium exposure category showed a difference of borderline significance (Wilcoxon's test: $p=0.05$, one tailed). The frequencies of arrhythmia when unexposed were somewhat higher than previously reported. Misclassification of the exposure and the possible confounding effect of physical workload and psychological strain may have obscured a causal relation and therefore a minor effect cannot be ruled out. The results do not support the notion that fluorocarbons induce cardiac arrhythmia in occupationally exposed refrigerator repairmen.
\end{abstract}

Flurocarbons have been used industrially since the 1930s, and are generally known under their commercial names such as Freon, Arcton or Frigen. In

Department of Occupational Medicine, University Hospital, Uppsala, Sweden

C Edling, G Ljungkvist

Department of Occupational Medicine, Örebro Medical Center Hospital, Óebro

C-G Ohlson, Á Oliv

Department of Clinical Physiology, Órebro Medical Center Hospital, Örebro

B Söderholm general, they are used as refrigerants, aerosol propellants, foam blowing agents, solvents, fire extinguishers, anaesthetics, and polymer intermediates. In total about 950000 metric tons are used annually in the world, of which about 5000 metric tons are used in Sweden. Considering the toxicity of fluorocarbons the cardiovascular system is the target organ in short term exposure. In experiments in mice and dogs FC 11 is the most toxic with regard to induction of tachycardia and cardiac arrhythmia. ${ }^{12}$

The information on human exposure is rather limited. Sudden deaths among aerosol sniffers have been attributed to cardiac arrhythmia induced by fluorocarbon propellants. ${ }^{3}$ A few exposure chamber studies have been conducted. Azar et al exposed volunteers to $10000 \mathrm{ppm}$ of FC 12 for 2.5 hours and found no cardiac arrhythmia. ${ }^{4}$ Stewart et al reported no changes in the electrocardiogram when healthy subjects were exposed to FC 11 and FC 12 with concentrations of 250,500 , and $1000 \mathrm{ppm}$ for periods of one minute to eight hours. ${ }^{5}$ Valic et al noticed that one minute of exposure to mixtures of FC 11 and FC 12 induced tachycardia and negative $T$ waves when the inhaled concentrations in air of the individual FCs were $16150 \mathrm{ppm}$ for FC 11 and $26800 \mathrm{ppm}$ for FC $12 .{ }^{6}$ This latter study suggests that there might be a hazard for occupationally exposed individuals, particularly refrigeration repairmen, since exposure concentrations may reach well above $10000 \mathrm{ppm}$.

Among the occupationally exposed are two reports on "palpitations"; Speizer et al reported that exposure to FC 22 caused palpitations at concentrations of $300 \mathrm{ppm}$ for two minutes among employees of a pathology department. ${ }^{7}$ The FC 22 was used in the preparation of frozen sections. Campbell et al found an increase of palpitations in refrigerator repair workers exposed to FC 22 and FC 11 . $^{8}$

There are no studies in which people have been followed up at their workplace. The aim of this study was to ascertain whether occupational exposure to fluorocarbons can induce cardiac arrhythmia in refrigerator repairmen.

\section{Material and methods}

STUDY GROUP

All shops engaged in repair and maintenance of refrigerators and cooling systems within four coun- 
ties in central Sweden were contacted and asked to take part in a health survey. About 350 workers were employed at the shops and 101 were eligible for participation in the survey. Twelve had to be excluded from the calculations owing to missing data or technical problems with the monitoring device. The remaining 89 constitute the final study group. They were all men with a mean age of 35 and had worked as refrigerator repairmen for 10 years on average.

\section{EXPOSURE}

The tasks of the subjects were to maintain and repair refrigerators in groceries, supermarkets, food industries, and homes. They could plan part of the work in advance but in the case of major problems with the cooling systems they had to rush off immediately several times a week. They were exposed to coolants from leaking compressors or from emptying or refilling the systems during the repairs.

All the coolants were fluorocarbons: FC 11, FC 12, FC 22, FC $500 \quad(73.8 \%$ FC $12,26.2 \%$ FC 152 a) and FC $502(48.8 \%$ FC $22,51.2 \%$ FC 115). Eighty nine per cent of the studied cooling systems contained FC 12 and 22 (table 1).

No information on the subjects' exposure to fluorocarbons in general was obtained. A questionnaire study of the exposure and working conditions of 248 repairers, including some of the participants in this survey, indicated that they spent $50-75 \%$ of their worktime directly working with various refrigerators and that exposure to fluorocarbons occurred only three to four times a week. Respirators were used only in case of irritating welding fumes.

For none of the subjects did the eight hour time weighted exposure exceed the Swedish threshold limit value, $500 \mathrm{ppm}$. The highest concentration was $280 \mathrm{ppm}$. Twenty four of the 89 subjects were exposed to concentrations higher than $750 \mathrm{ppm}$ in 15 minutes, the Swedish short term exposure limit: $750 \mathrm{ppm}$ was exceeded at any time during the monitoring periods by 62 subjects and the highest concentration recorded was 19300 ppm corresponding to a mean value in one minute of $14000 \mathrm{ppm}$.

\section{DESIGN}

The main parameter in this study was the frequency of arrhythmia. The design was based on the assumptions that a possible induction of cardiac arrhythmia caused by fluorocarbons occurs almost immediately after exposure starts, as the substance concentrates in

Table 1 Frequency distribution of the different fluorocarbons measured during the monitoring

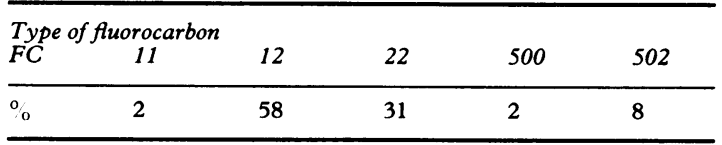

the heart muscle, and that the disturbance declines shortly after the end of exposure as the concentration begins to decrease. Such an effect on the heart should be reflected in higher frequencies of arrhythmia in exposed than in unexposed periods.

The exposure during a typical workday occurred during rather short periods and it was possible to collect data for both exposed and unexposed periods in the same subject. Consequently, a within subject comparison was applied and a reference group was not necessary. Moreover, the statistical analyses were simplified since the effects of most moderators or confounding factors were negligible.

\section{MEASUREMENT: INDEPENDENT VARIABLE}

The subjects were monitored in their regular work by measuring the concentrations of fluorocarbons in the breathing zones and by simultaneous recording of cardiac activity. The monitoring covered part of one workday or, in some subjects, a whole workday. Tasks with high probability of exposure to fluorocarbons were selected for monitoring. Four subjects were not exposed to any detectable concentrations of flurocarbons during the monitoring period.

The exposure concentrations of fluorocarbons in the breathing zone were measured by two experienced industrial hygienists using a direct recording infrared spectrometer (MIRAN). A flexible hose was attached to the breathing zone and the air was pumped continuously through the instrument without interfering with the work. The data were stored in a data logger to be processed later by a personal computer. Before each monitoring period the instrument was calibrated for the relevant fluorocarbons.

The individual exposure data were displayed on a graph indicating both the instantaneous level and the extact time. From these graphs the time periods with different levels of exposure could be identified.

\section{MEASUREMENT: DEPENDENT VARIABLE}

The recording of the heart activity was carried out by trained nurses who used an ambulatory Tracker Reinholds Medical to record the electrocardiograms. The time of the electrocardiogram was synchronised exactly with that of the fluorocarbon measuring device.

The tapes were analysed by a Digital PDP II after scanning with Reinholds medical replay II high speed ECG analyser. All suspected abnormalities were printed on electrocardiographic paper and reviewed by a cardiologist (BS) without knowledge of the exposure concentrations.

Two types of arrhythmia were recorded, ectopic beats and sudden bradycardias. Ectopic beats referred both to supraventricular and ventricular ectopic beats. Sudden bradycardia referred to inhibition of the normal pace rate of the sinus node 
regardless of whether caused by increased vagal tone or by block of the atrioventricular conduction system.

Exposure to fluorocarbons could occur at any time during the monitoring period. In some cases there was one single and long exposure period covering about half the monitoring period but in most cases there were two or more short exposure periods. The mean time in exposure $\geq 750 \mathrm{ppm}$ was 11 minutes and the mean time in $<750 \mathrm{ppm}$ was 174 minutes, yielding about three hours of total monitoring time on average.

The study group was divided into four exposure categories according to the following criteria:

High exposure $>750 \mathrm{ppm}>10$ minutes and peaks $>5000 \mathrm{ppm}$

Medium exposure $>750 \mathrm{ppm}$ 5-10 minutes and peaks $3000-5000 \mathrm{ppm}$

Low exposure $>750 \mathrm{ppm}<5$ minutes and peaks $<3000 \mathrm{ppm}$

Minimal exposure $<750 \mathrm{ppm}$

The criterion of $750 \mathrm{ppm}$ was based on the Swedish short term exposure limit. Four subjects had no detectable exposure and were excluded from the calculations.

\section{DATA ANALYSIS}

Cardiac arrhythmia tend to occur paroxysmally rather than be evenly distributed in time. The severeness of an arrhythmia is probably not proportional to the number of ectopic beats. To make the frequency distribution less skewed it therefore seemed justified to count time units-that is, minutes containing arrhythmias-regardless of the number of ectopic beats within that time. This will be referred to as "arrhythmia minutes." The frequency of arrhythmia minutes during an exposed period was then compared with the same frequency during the rest of the monitoring, the unexposed period. The comparison was based both on the absolute differences and the relative differences (as per cent of the unexposed frequencies).

Cardiac exposure-that is, the concentration of flurocarbons in the heart not in the breathing zoneis the relevant exposure variable of this study. Unfortunately, this concentration cannot be calculated due to a lack of information on the biotransformation of the fluorocarbons. Animal experiments indicate however, that the uptake in the blood and transportation to the heart muscle takes place within minutes. Since there was a delay of less than one minute in the measuring instrument due to the flow time in the hose, we considered the cardiac exposure to begin one minute after exposure to fluorocarbons as indicated on the measuring instrument.

Although the half life of fluorocarbons in the heart muscle is unknown, a crude estimate may be obtained based on information on the half life in blood and the assumed distribution coefficient between heart and blood.

Accordingly, we assumed that the concentrations of fluorocarbons in the human heart decreased to half or less within half an hour after a single peak exposure. Biologically significant cardiac exposure was estimated to cease within 30 minutes-one hour on average after cessation of exposure-that is, when the measuring instrument indicated no fluorcarbons. The corresponding time after a single and short exposure peak was estimated to be about 30 minutes.

To sum up, the criterion for the period of cardiac exposure was one minute after exceeding $750 \mathrm{ppm}$ as indicated on the measuring instrument and $45 \mathrm{~min}$ utes after the fall below this level or 30 minutes after a peak exposure for the high, medium, and low exposure categories. The corresponding periods of the minimal exposure category were assessed by eye directly from the graph.

Even though the frequency distribution of arrhythmia is strongly skewed, the intraindividual differences between exposed and unexposed periods may be expected to be normally distributed. A graphical plot of the data showed a strong concentration of the data around \pm 0 and long tails. Therefore, the statistical significance of the absolute differences between the frequencies of arrhythmia minutes in the exposed and unexposed periods was tested by use of a non-parametric test, Wilcoxon signed rank test. The test was carried out both for the whole study group and after stratification for exposure category. The mean pulse rates of the exposure categories were compared by use of Student's $t$ test.

\section{Results}

No appreciable difference between exposed and unexposed frequencies of ectopic beats was observed for the whole study group (table 2). No clear dose effect relation could be shown for the same differences after stratification for exposure category. The high exposure category had low mean frequencies of ectopic beats and the absolute difference was correspondingly low, although the relative difference was rather high, $63 \%$. The absolute difference for the medium exposure category was 0.019 and was statistically of borderline significance $(p=0.05$, one tailed). The corresponding differences for the high and the medium exposure categories together was also just of borderline significance $(p=0.05$, one tailed). The low and minimal exposure categories had lower exposed than unexposed frequencies corresponding to relative differences of $-15 \%$ and $-5 \%$ respectively. The differences were not statistically significant $(p>0.05)$.

A similar pattern was observed for the differences in frequencies of sudden bradycardia after stratification for exposure category (table 3 ). The absolute difference for the high and the medium exposure 
Table 2 Mean frequencies of minutes with ectopic beats during exposed and unexposed periods and the mean intraindividual differences between exposed and unexposed frequencies for different exposure categories

\begin{tabular}{lrllll}
\hline \multirow{5}{*}{ Exp category } & No & Exposed & Unexposed & Difference & $(\%)$ \\
\cline { 3 - 6 } & & & & & \\
\hline High & 5 & 0.013 & 0.008 & 0.005 & $(63)$ \\
Medium & 14 & 0.096 & 0.077 & $0.019^{\star}$ & $(25)$ \\
Low & 43 & 0.070 & 0.084 & -0.013 & $(-15)$ \\
Minimal & 23 & 0.072 & 0.076 & -0.004 & $(-5)$ \\
All & 85 & 0.071 & 0.076 & -0.005 & $(-7)$ \\
\hline
\end{tabular}

${ }^{\star} p=0.05$, one tailed.

categories together was 0.034 corresponding to a relative difference of $58 \%$. This difference was statistically of borderline significance ( $p$ just below 0.05 , one tailed; $p>0.05$, two tailed). None of the other differences was statistically significant.

The calculations were also carried out with the information on the concentrations in the breathing zone without time correction-that is, without consideration of the estimated cardiac exposure. The outcome was identical to that obtained with time correction. Furthermore, the mean pulse rates during exposed periods for the different exposure categories were compared. The medium exposure category had a slightly higher mean pulse rate than the other categories but the difference was not statistically significant.

It was not possible to study the impact of the different types of fluorocarbons. Subjects with medium or high exposure to FC 22 seemed to have the highest absolute differences between exposed and unexposed frequencies of ectopic beats but the difference was still just of statistical borderline significance $(p=0.05)$.

Six men had peak exposures exceeding $10000 \mathrm{ppm}$. Four were exposed to FC 12 (11 400, 13800,13900 , and $16500 \mathrm{ppm}$ ) and two to FC 22 (12 900 and $19300 \mathrm{ppm}$ ). Among these six, the subject exposed to the lowest peak of FC 12 had an ectopic beat within eight minutes after the peak exposure and no arrhythmia when unexposed. For

Table 3 Mean frequencies of minutes with sudden bradycardia during exposed and unexposed periods and the mean intraindividual differences between exposed and unexposed frequencies for different exposure categories

\begin{tabular}{lrlllr}
\hline & \multicolumn{5}{c}{ Mean frequencies } \\
\cline { 3 - 6 } Exp category & No & Exposed & Unexposed & Difference & $(\%)$ \\
\hline High & 5 & 0.034 & 0.024 & 0.010 & $(42)$ \\
Medium & 14 & 0.113 & 0.071 & $0.042 \star$ & $(59)$ \\
Low & 43 & 0.067 & 0.090 & -0.023 & $(-26)$ \\
Minimal & 23 & 0.026 & 0.036 & -0.010 & $(-28)$ \\
All & 85 & 0.062 & 0.068 & -0.007 & $(-10)$ \\
\hline
\end{tabular}

${ }^{\star} p<0.05$, one tailed; $p>0.05$, two tailed. High and medium exposure categories put together. the other five there were no differences between the exposed and unexposed periods with regard to arrhythmia.

The possible impact of physical workload on the occurrence of arrhythmia was investigated by comparing the work tasks of subjects with high absolute differences of frequencies of arrhythmia and subjects without arrhythmia during exposed time. The selection criteria were arbitrarily set at the extremes to yield a limited number of subjects and 13 subjects were accordingly selected from both groups. Their work tasks while exposed were checked through notes made during the exposure measurements and classified according to physical workload. There was no significant difference between the groups.

\section{Discussion}

This field study of refrigerator repairmen did not show a consistent effect of exposure to fluorocarbons on the occurrence of cardiac arrhythmia. The medium exposure category showed the highest positive differences in ectopic beats and sudden bradycardia between exposed and unexposed periods $(p=0.05)$.

The design of the study, a within subject comparison, eliminated the most common confounding factors relevant to the occurrence of cardiac arrhythmia and justified the adoption of a simple statistical model. The statistical power of the study, the ability to discover a certain effect with a reasonably high probability, using a non-parametric statistical model, may be indirectly estimated by use of the Student's $t$ test. The Wilcoxon signed rank test has a relative efficiency of 0.96 compared with the $t$ test. ${ }^{9}$ A prerequisite for a power calculation based on the $t$ test is knowledge of the standard deviation. Due to the non-normal distribution of the frequency parameter the standard deviations were not calculated. Cardiac arrhythmia occurred frequently, however, and therefore the sample size seems to give sufficient power to detect a major effect of the fluorocarbons.

The frequency of arrhythmia was generally surprisingly high, regardless of exposure. Sixty five subjects $(73 \%)$ had one or more ectopic beats and 70 $(79 \%)$ one or more episodes of sudden bradycardia. Twice as many subjects had supraventricular than ventricular ectopic beats. The average number of ventricular ectopics beats in one hour was higher than that of the supraventricular ectopic beats, 8.6 v $2 \cdot 0$. If single and minor arrhythmic events are disregarded only 17 subjects were free from arrhythmia. The 90th percentile in the distribution of mean number of ectopic beats an hour was 13 ectopic beats in one hour.

The question is whether the frequency of arrhythmia among the refrigerator repairmen in this study can be regarded as normal or increased? Published 
studies on ambulatory electrocardiographic monitoring of healthy men younger than 65 are scanty and the number does not seem to exceed ten. ${ }^{111} \mathrm{~A}$ study of the frequency of ectopic beats among 147 actively employed healthy men aged from 15 to 65 showed that $25 \%$ had no supraventricular and $54 \%$ no ventricular ectopic beats in 24 hours. The 90th percentile was 10.3 in subjects aged $15-66 .{ }^{10}$ In healthy subjects ventricular ectopic beats have been detected in between $17-80 \%$ depending on the recording time duration and the characteristics of the population examined. ${ }^{11}$

Although data from other studies cannot be directly compared due to different measures, the refrigerator repairmen in our study had a seemingly somewhat higher prevalence of ectopic beats than generally oberved. One possible explanation is that the repairmen were under more psychological strain, due to the demands of the work tasks, than other studied populations. The mean pulse rate was high, which supports the impression of psychological strain. Another possibility could be that long term exposure to fluorocarbons induce chronic effects on the heart. There are, however, despite a number of animal experiments, no data supporting this and, in addition, a cohort study of refrigerator repairmen did not show any increased mortality due to cardiovascular diseases. ${ }^{12}$

Some misclassification of exposure could not be avoided in this study. Firstly, the limits of the exposure categories were set rather arbitrarily as there is no a priori knowledge about cardiotoxic concentrations of fluorocarbons in man. Unexposed periods were not defined uniformly because parts of the periods with concentrations less than $750 \mathrm{ppm}$ were classified as unexposed in the high, medium, and low exposure categories but classified as exposed in the minimal exposure category.

Secondly, the classification of the individual graphs were performed by eye and no absolute criteria could be applied to the graphs, which showed great variations in the number and duration of the exposure peaks. The classifications were, however, performed without knowledge of the arrhythmia frequencies and may therefore be regarded as nondifferential-that is, not causing any bias. Furthermore, the subjects were exposed to different fluorocarbons and the study population was too small to justify separate analyses by type of flurocarbon. In animal experiments FC 11 has been the most toxic with regard to inducing cardiac arrhythmia ${ }^{12}$ but in our study only two subjects were exposed to FC 11 . Neither had any increase in the number of arrhythmia during exposure compared with the unexposed periods.

The dependent variable, the frequency of arrhythmia, was reduced to decrease the extreme skewness. This variable transformation should not have decreased the sensitivity of this measure.
Physical stress is a factor related to the specific worktasks of the subjects and may be a confounding factor. If the subjects performed strenuous work in extreme body positions while emptying the cooling systems, and hence while being highly exposed, such physical stress might have caused ectopic beats or alteration in the pulse rate. Physical stress could not be adjusted for in the calculations even though the comparison of subjects with high absolute differences of frequencies of arrhythmia and subjects without arrhythmia while exposed did not indicate such an effect.

This is, to our knowledge, the first field study where workers exposed to fluorocarbons have been monitored with an electrocardiogram during work. Consequently, the results cannot be compared and discussed in relation to other field studies. Experimentally, however, cardiac effects have been reported in several species, including man. Most workers in this study were exposed to FC 12 and FC 22. There are some human data with regard to FC 12 . In a case report ECG abnormalities (tachycardia with supraventricular extrasystoles and a high frequency of monofocal extrasystoles) were associated with exposure to FC $12 .{ }^{13}$ Neither exposure time nor level at the time of illness was reported. Exposure chamber studies have not shown any ECG abnormalities when volunteers have been exposed to FC 12 from 250 up to $10000 \mathrm{ppm}$ for 2.5 hours. $^{45}$

Taking these results and our own data into consideration it might be suggested that there is no obvious risk for heart effects when exposure concentrations of FC 12 are low, well below $10000 \mathrm{ppm}$. On the other hand, higher concentrations could induce tachycardia, ${ }^{6}$ and our findings of a possible effect among the medium exposed are not totally inconsistent with animal and human data. Nevertheless, the absence of a dose effect pattern and the lack of a relation between peak exposures (over $10000 \mathrm{ppm}$ ) and arrhythmia do not favour a causal relation.

With respect to FC 22, animal data indicate that this substance has a somewhat lower cardiotoxic effect than FC 12 but human data are sparse. ${ }^{14} \mathrm{~A}$ report by Speizer et al states that exposure to 300 ppm of FC 22 causes palpitations. ${ }^{7}$ Our results do not show such low exposure concentrations to have any adverse effects on heart activity. One finding from a questionnaire survey we conducted among 248 refrigerator repairmen was that the reported frequency of palpitations was $12 \%$ which was not higher than in non-exposed reference groups.

Misclassification of the exposure variable may obscure a causal relation and therefore a minor effect cannot be ruled out. Such an interpretation is supported by the fact that subjects with high and medium exposure showed an increased frequency of arrhythmia while exposed. Physical strain may have 
acted, however, in either direction as a confounding factor. The results of this study do not indicate that fluorocarbons induce cardiac arrhythmia among occupationally exposed refrigerator repairmen.

In summary, whereas no final conclusions concerning the risk of cardiac arrhythmia due to short and moderately high exposures to fluorocarbons can be drawn from this study, a conservative interpretation of the data is that no consistent associations have been shown.

We gratefully appreciate the cooperation of Ann Ekerljung, Eva Falkner, Birgitta Fält, Siv Lunander, Åda Lundin, and Margeretha Torgén for carrying out the ambulatory electrocardiograms at the places of work. We thank Mrs Christina Söderqvist for typing the manuscript. The project was supported by the Swedish Work Environment Fund.

1 Aviado DM, Belej MA. Toxicity of aerosol propellants on the respiratory and circulatory systems. 1. Cardiac arrhythmias in the mouse. Toxicology 1974;2:31-42.

2 Belej MA, Aviado DM. Cardiopulmonary toxicity of propellants for aerosols. J Clin Pharmacol 1975;15:105-15.

3 Bass M. Sudden sniffing death. JAMA 1970;212:2075-9.

4 Azar A, Reinhardt CF, Maxfield ME, Smith PE, Mullin LS.

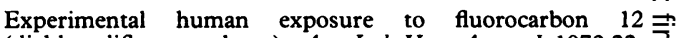
(dichlorodifluoromethane). Am Ind Hyg Assoc J 1972;33: ज़ 207-16.

5 Stewart RD, Newton PE, Baretta ED, Hermann AA, Forster HV, Soto RJ. Physiological response to aerosol propellants. 흐 Environ Health Perspect 1978;26:275-85.

6 Valic F, Skuric Z, Bantic Z, Rudar M, Hecej M. Effects of $\frac{\sigma}{T}$ fluorocarbon propellants on respiratory flow and ECG. $B r J \stackrel{\Phi}{\Phi}$ Ind Med 1977;34:130-6.

7 Speizer FE, Wegman DH, Ramirez A. Palpitation rates associated with fluorocarbon exposure in a hospital setting. $N \vec{O}$ Engl J Med 1975;292:624-6.

8 Campbell DD, Lockey JE, Petajan J, Gunter BJ, Rom WN. Health effects among refrigeration repair workers exposed to $\vec{\omega}$ fluorocarbons. $\mathrm{Br} J$ Ind Med 1986;43:107-11.

9 Armitage P, Berry G. Statistical methods in medical research. 2nd $O$ ed. Oxford: Blackwell Scientific Publications, 1987.

10 Orth-Gomer K, Hogstedt C, Bodin L, Söderholm B. Frequency of extrasystoles in healthy male employees. $\mathrm{Br}$ Heart $\mathrm{J}$ 1986;55:259-64.

11 Green MS, Jucha E, Luz J, Cocos M, Nurynberg M, Rosenberg $\omega$ N. Prevalence and correlates of ventricular premature beats $N$ detected by ambulatory electrocardiography in working populations. Am J Epidemiol 1988;127:955-68.

12 Szmith M, Axelson O, Edling C. Acta Societatis Medicorum 은 Svecanae Hygiea 1981;90:77. (In Swedish.)

13 Åström T, Jonsson A, Järvholm B. Exposure to fluorocarbons $>$ during the filling and repair of air-conditioning systems in cars-a case report. Scand J Work Environ Health 1987; $\bar{\Omega}$ 13:527-8.

14 Reinhardt CF, Azar A, Maxfield ME, Smith PE, Mullin LS Cardiac arrhythmias and aerosol "sniffing". Arch Environ Health 1971;22:265-79.

Accepted 5 May 1989 